\title{
Ursodeoxycholic Acid at 18-22 mg/kg/d Showed a Promising Capacity for Treating Refractory Primary Biliary Cholangitis
}

\author{
Xinyu Xiang, ${ }^{1}$ Xiaoli Yang, ${ }^{2}$ Mengyi Shen, ${ }^{1}$ Chen Huang, ${ }^{1}$ Yifeng Liu, ${ }^{1}$ Xiaoli Fan $\left(\mathbb{D},{ }^{1}\right.$ \\ and Li Yang (iD) 1 \\ ${ }^{1}$ Department of Gastroenterology and Hepatology, \\ Sichuan University-University of Oxford Huaxi Joint Centre for Gastrointestinal Cancer, West China Hospital, \\ Sichuan University, Chengdu, China \\ ${ }^{2}$ Department of Gastroenterology \& Hepatology, West China Hospital, Sichuan University, Chengdu, Sichuan 610041, China
}

Correspondence should be addressed to Li Yang; yangli_hx@scu.edu.cn

Received 5 December 2020; Revised 13 January 2021; Accepted 15 January 2021; Published 21 January 2021

Academic Editor: Alessandro Granito

Copyright (c) 2021 Xinyu Xiang et al. This is an open access article distributed under the Creative Commons Attribution License, which permits unrestricted use, distribution, and reproduction in any medium, provided the original work is properly cited.

\begin{abstract}
Aim. To compare the response between the current recommended dosage $13-15 \mathrm{mg} / \mathrm{kg} / \mathrm{d}$ and $20 \mathrm{mg} / \mathrm{kg} / \mathrm{d}$ dose of ursodeoxycholic acid (UDCA) in primary biliary cholangitis (PBC) patients who do not respond completely to a standard dose of UDCA. Methods. We included 73 patients with poor response and randomized them into two groups to investigate whether increasing the dosage of UDCA was beneficial to nonresponders. Patients assigned to the $13-15 \mathrm{mg} / \mathrm{kg} / \mathrm{d}$ group continued with standard therapy, and participants in the $18-22 \mathrm{mg} / \mathrm{kg} / \mathrm{d}$ group switched to the higher dosage (18-22 mg/kg/d), with a follow-up of $12 \mathrm{months}$ for both groups. The primary endpoints were the rate of response at 6 months and drug side effects. Results. According to the Paris 2 criteria, patients receiving $18-22 \mathrm{mg} / \mathrm{kg} / \mathrm{d}$ UDCA achieved a response rate of $59.4 \%$ compared with $36.1 \%$ in the standard dosage group $(P=0.046)$ at 6 months, respectively. At 12 months, the high-UDCA-dosage group achieved a response rate of 59.4\% compared with $47.2 \%$ in the standard dosage group $(P=0.295)$, respectively. Additionally, the risk score predicted by the UKPBC model was lower in high-dosage UDCA-treated patients than in the standard dosage group (all $P<0.05$ ). Side effects include diarrhea, nausea and vomiting, rash, and newly developed high blood pressure, which were mild and tolerated. Conclusions. Patients treated with the high UDCA dosage showed some advantages over those who continued the standard dosage in terms of biochemical remission and disease progression, indicating that standard therapy with UDCA for 6 months and then another 1 year with high UDCA dosage for nonresponders could be a treatment option before second-line therapy is recommended.
\end{abstract}

\section{Introduction}

Primary biliary cholangitis (PBC), an autoimmune disease predominantly affecting females, is characterized by clinical manifestations of progressive cholestasis, chronic elevation of alkaline phosphatase (ALP), and positive antimitochondrial antibodies (AMAs) [1]. Liver biopsy typically presents as nonsuppurative destructive cholangitis, and histological evidence is required only when antibodies are absent $[2,3]$. Untreated patients can develop cirrhosis, hepatic failure, and liver-related death, and lifelong medication use is strongly recommended [4].
Ursodeoxycholic acid (UDCA) has been recommended as the first-line therapy for PBC patients according to guidelines since its approval by the Food and Drug Administration (FDA) in 1977, as it benefits biochemical mitigation and transplantation-free survival $[5,6]$. UDCA was also found to slow the patient's histological progress compared with a placebo [7]. Studies have revealed quantitative and compositional changes in the bile pool of $\mathrm{PBC}$ patients, and a positive correlation was found between the response and enrichment of UDCA in the patient's bile pool [8].

Despite the efficacy, it was estimated that nearly $40 \%$ of PBC patients on standard UDCA therapy did not achieve a 
complete response [9]. Many clinical trials have been carried out to explore alternative options for those nonresponders, one of which was an increased dosage of UDCA. A prior clinical trial comparing the effectiveness between $20 \mathrm{mg} / \mathrm{kg} /$ $\mathrm{d}$ and $10 \mathrm{mg} / \mathrm{kg} / \mathrm{d}$ UDCA found that the $20 \mathrm{mg} / \mathrm{kg} / \mathrm{d}$ dosage resulted in greater biochemical decreases [10], yet no trials were conducted to compare the response between the current recommended dosage $13-15 \mathrm{mg} / \mathrm{kg} / \mathrm{d}$ and $20 \mathrm{mg} / \mathrm{kg} /$ d. Therefore, we performed this study to investigate the efficacy and safety of UDCA at a dosage of $18-22 \mathrm{mg} / \mathrm{kg} / \mathrm{d}$ in treating PBC patients with a poor response.

\section{Methods}

2.1. Study Population. Patients with a recent or previous diagnosis of PBC were recruited at the inpatient and outpatient clinic of the Gastroenterology and Hepatology Department, West China Hospital. PBC was diagnosed when at least two of the following three criteria were fulfilled: (1) serum alkaline phosphatase (ALP) levels at least 1.5 times the normal upper limit for more than 6 months; (2) the presence of AMAs in serum; and (3) representative histological manifestations of portal area inflammation and bile duct injury [11]. After 6 months of standard treatment, patients with an incomplete response, which was defined as the Paris 2 criteria [12] (a serum level of ALP or aspartate aminotransferase $($ AST $)>1.5$ times the upper limit of the normal range or an abnormal total bilirubin (TB) level), were eligible for entry. The inclusion criteria were patients diagnosed with PBC who were treated with $13-15 \mathrm{mg} / \mathrm{kg} / \mathrm{d}$ UDCA at West China Hospital for 6 months and obtained a suboptimal response to UDCA. The exclusion criteria included diseases that are easily confused with PBC, e.g., autoimmune hepatitis and primary sclerosing cholangitis. Cirrhosis was evaluated according to the liver histology, abdominal imaging, such as ultrasonography (US), computed tomography (CT), and/or magnetic resonance imaging (MRI), or endoscopic examination [13]. Patients with decompensated cirrhosis were excluded, which was diagnosed by the presence of clinical complications [14].

Patients underwent autoimmune investigations: AMA, anti-Sp100 and anti-gp210 testing by immunoblotting (IB), and indirect immunofluorescence, searching for PBC-specific ANAs. All parameters were examined in the Department of Laboratory Medicine of West China Hospital, which was certified by the College of American Pathologists (CAP). Some cases underwent investigations for anti-Sp100 and anti-gp210, which were carried out by the third party (Kindstar Global Corporation, China).

2.2. Study Design. For this study, we conducted a prospective, randomized, open-label pilot trial to investigate whether a higher dosage of UDCA therapy could benefit PBC patients and per-protocol analysis was applied. All recruited patients were given $13-15 \mathrm{mg} / \mathrm{kg} / \mathrm{d}$ UDCA at the time of diagnosis according to the current guidelines [15]. They were required to complete a serum biochemical blood test at 6 months. Patients who did not meet the suggested response of the Paris 2 criteria were considered nonresponders. After confirmation of eligibility, we randomly allocated participants to receive a higher dosage of UDCA (18-22 mg/kg/d) or to continue with the previous $13-15 \mathrm{mg} /$ $\mathrm{kg} / \mathrm{d}$ UDCA therapy. The drug side effects observed included diarrhea, nausea and vomiting, rash, and new onset of high blood pressure [16]. The response was evaluated in the two groups at 6 months and 12 months. Prognostic evaluation was determined by the established UK-PBC score and Globe score [17].

Assuming a dropout rate of $10 \%$, it was estimated that a sample of 50 patients was needed with a projected response rate of $50 \%$ in the high-dosage group and $10 \%$ in the standard group to have $90 \%$ power with a significant difference at a value of $\alpha=0.05$. This study was registered at ClinicalTrials.gov (identifier: NCT03345589), and all patients provided written informed consent.

2.3. Outcomes. The primary outcome was the percentage of patients with a complete biochemical response as defined by the Paris 2 criteria and side effects. The UK-PBC scoring system was used to detect the anticipated 5-, 10-, and 15-year risks of liver transplantation, and liver-related fatality and Globe scores were determined to predict transplant-free survival. The UK-PBC score incorporates the baseline platelet count and serum albumin level and the serum bilirubin, transaminases, and ALP levels measured after 12 months of UDCA treatment [17]. Secondary outcomes included changes from baseline in serum TB, ALP, AST, alanine aminotransferase, $\gamma$-glutamyltransferase (GGT), albumin, globulin, immunoglobulin $\mathrm{M}$, and so on; liver fibrosis was measured by noninvasive indices, including the AST-to-platelet ratio index (APRI) and the fibrosis index based on four factors (FIB-4). The formula used for calculating FIB-4 equates to age (years)xAST (U/L)/(platelets $\left.\left[10^{9} / \mathrm{L}\right] \mathrm{x}(\mathrm{ALT}[\mathrm{U} / \mathrm{L}])^{1 / 2}\right)[18]$; we also compared the response difference by definition of the Barcelona criteria because patients had been on medication at 6 months and 1 year (Barcelona criteria: ALP decrease $>40 \%$ of pretreatment levels or normalization), respectively.

2.4. Statistical Analysis. A chi-square test was performed to compare the differences in the percentage of patients with a complete biochemical response in the two groups. Comparisons of baseline data, UK-PBC scores, APRI, and FIB-4 values were performed using the Mann-Whitney $U$ test. Continuous variables are expressed as medians with interquartile range. Two-sided $P$ values of less than 0.05 were considered statistically significant. The statistical analysis was performed using SPSS 24.0 (SPSS version 24.0 for Windows; IBM Corp, Armonk, NY, USA).

\section{Results}

Our trial was conducted following the procedure steps demonstrated in Figure 1. Patients at entry exhibited no differences in serum biochemical markers (Table 1). More than $90 \%$ of the population in both groups consisted of 


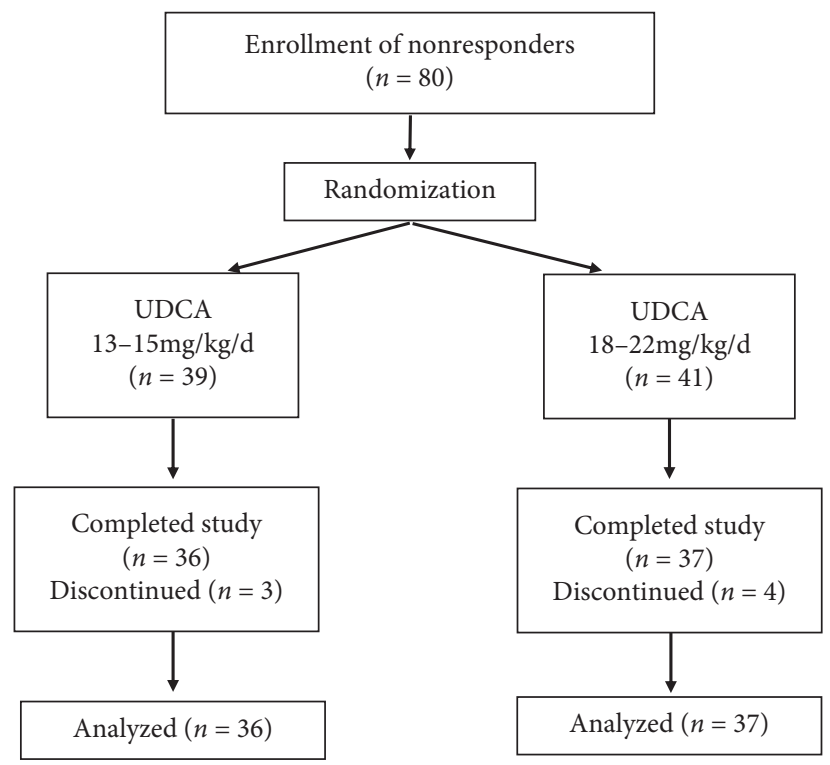

Figure 1: Flowchart of the trial.

TABLE 1: Characteristics of study population at entry.

\begin{tabular}{|c|c|c|c|}
\hline & $\begin{array}{c}13-15 \mathrm{mg} / \mathrm{kg} / \mathrm{d} \\
(n=36)\end{array}$ & $\begin{array}{c}18-22 \mathrm{mg} / \mathrm{kg} / \mathrm{d} \\
(n=37)\end{array}$ & $P$ value \\
\hline Age, year & $52.0(46.0,58.8)$ & $52.0(46.5-56.0)$ & 0.623 \\
\hline Female, no. (\%) & $30(83.3 \%)$ & $33(89.2 \%)$ & 0.476 \\
\hline Fatigue (\%) & $11 / 36(30.6 \%)$ & $13 / 37(35.1 \%)$ & 0.677 \\
\hline Pruritus (\%) & $18(50.0 \%)$ & $19(51.4 \%)$ & 0.995 \\
\hline PLT, $10^{9} / \mathrm{L}$ & $170.0(102.3,234.5)$ & $181.0(137.5,235.5)$ & 0.318 \\
\hline $\mathrm{TB}, \mu \mathrm{mol} / \mathrm{L}$ & $17.4(14.1,27.3)$ & $15.3(11.2,22.4)$ & 0.072 \\
\hline ALP, IU/L & $281.0(216.0,394.5)$ & $238.0(203.0,338.0)$ & 0.156 \\
\hline AST, IU/L & $58.5(45.0,94.0)$ & $54.0(42.5-67.0)$ & 0.180 \\
\hline $\mathrm{ALT}, \mathrm{IU} / \mathrm{L}$ & $64.0(40.5,77.0)$ & $53.0(44.0,78.0)$ & 0.938 \\
\hline $\mathrm{ALB}, \mathrm{g} / \mathrm{L}$ & $43.3(39.7,47.5)$ & $45.4(43.2,46.6)$ & 0.133 \\
\hline GLB, g/L & $36.7(31.3,39.2)$ & $34.3(30.6,35.6)$ & 0.090 \\
\hline GGT, IU/L & $220.0(123.3,288.0)$ & $161.0(110.5-261.5)$ & 0.227 \\
\hline IGM, mg/L & $3145.0(1787.0,4265.0)$ & $3000.0(2090.0,3815.0)$ & 0.728 \\
\hline $\mathrm{CHOL}, \mathrm{mmol} / \mathrm{L}$ & $5.3(4.4,6.9)$ & $6.0(5.3-6.4)$ & 0.348 \\
\hline APRI & $1.17(0.64,1.18)$ & $0.97(0.61,1.27)$ & 0.120 \\
\hline FIB-4 & $3.02(1.49,5.62)$ & $2.14(1.41,3.00)$ & 0.085 \\
\hline AMA, no. (\%) & $22(61.1 \%)$ & $29(78.3 \%)$ & 0.108 \\
\hline MND (\%) & $0(0 \%)$ & $1(2.7 \%)$ & $>0.999$ \\
\hline Anti-Sp100* & $0 / 5(0 \%)$ & $1 / 7(14.3 \%)$ & $>0.999$ \\
\hline RL/M (\%) & $11(30.6 \%)$ & $12(32.4 \%)$ & 0.863 \\
\hline Anti-gp210* & $1 / 5(20.0 \%)$ & $2 / 7(28.6 \%)$ & $>0.999$ \\
\hline Liver cirrhosis, no. (\%) & $12(33.3 \%)$ & $7(18.9 \%)$ & 0.181 \\
\hline
\end{tabular}

* Only 12 cases in our trial underwent investigations for anti-Sp100 and anti-gp210 by immunoblotting. Note. PLT, platelet count; TB, total bilirubin; ALP, alkaline phosphatase; AST, aspartate aminotransferase; ALT, alanine aminotransferase; ALB, albumin; GLB, globulin; GGT, $\gamma$-glutamyltransferase; IGM, immunoglobulin M; CHOL, cholesterol; PT, prothrombin time; APRI, aspartate aminotransferase/platelet ratio index; FIB-4, fibrosis index based on the four factors; AMA, antimitochondrial antibody; MND, multiple nuclear dots; RL/M, rim-like/membranous.

females in their 50s. No differences were found in the percentage of fatigue and pruritus when enrolled in the study ( $35.1 \%$ vs. $30.6 \%$ and $51.4 \%$ vs. $50.0 \%$, respectively). $61.1 \%$ of the population in the standard group and $78.3 \%$ in the highdosage group was AMA positive. Multiple nuclear dot (MND) and rim-like/membranous antinuclear (RL/M) antibodies were rare findings in our study group, and only 12 cases in our study underwent investigations for anti-Sp100 and anti-gp210 by immunoblotting. No differences were found in assessments of degree of fibrosis according to the APRI and FIB-4 values and the percentage of cirrhosis between the standard and high-dosage groups at baseline.

At 6 months, response rates assessed by the Paris 2 criteria were significantly different between the two groups, 
with $59.4 \%$ of patients achieving a complete response in the high-dosage group compared with $36.1 \%$ in the standard group $(P=0.046)$. In contrast, under the Barcelona criteria, the high-dosage group did not show a significant increase in the proportion of responders $(P=0.483)$ (Table 2$)$. At 12 months, there was no difference in the response rates between the two groups using Paris 2 and Barcelona criteria, respectively (Table 3 ). The UK-PBC score at 12 months showed a lower incidence of severe liver-related events in the $18-22 \mathrm{mg}$ group than in the group continuing with the standard UDCA dosage (Table 4). APRI and FIB-4 scores of high-dosage patients were lower than that of the standard dosage group at 6 and 12 months (all $P<0.05$ ) (Table 5).

Overall, changes from baseline in the levels of critical biomarkers of $\mathrm{PBC}$ were partly consistent with the primary outcome. The reduction rate of ALB and PLT in the highdosage group was higher than that of the standard group at 6 months (Table 6). However, the reduction rate of the levels of these critical biomarkers was similar in both groups at 12 months (Table 7).

To assess safety, side effects of UDCA, including diarrhea, nausea and vomiting, rash, and newly developed high blood pressure, were evaluated. Two patients in the highdosage group developed mild diarrhea, which was tolerable. Generally, both dosages were well tolerated by patients with no occurrence of serious adverse reactions. More detailed information is presented in Table 8 .

\section{Discussion}

The primary outcomes suggested that a dosage of $18-22 \mathrm{mg}$ was superior to $13-15 \mathrm{mg}$ in treating PBC nonresponders when measured by the Paris 2 criteria. It is reasonable to draw the conclusion that an $18-22 \mathrm{mg}$ dosage is more effective than standard therapy in these conditions. The UKPBC score predicted the advantage of 18-22 mg of UDCA in preventing liver-related adverse events and decreasing the risk of liver transplantation. Also, lower APRI and FIB-4 indices in high-dosage patients indicated that increasing the dosage might delay the development of hepatic cirrhosis.

PBC-specific ANAs are associated with severe PBC and their presence may also predict a less response to UDCA treatment $[19,20]$. In our study population, MND and RL/M antinuclear antibodies were rare findings, which implied a favourable course. According to the Paris 2 criteria, the biochemical response was assessed a year after taking the standard dosage of UDCA. However, an early identification was found by Zhang et al. and measurements at six months may be used instead of measurements at 1 year because these values exhibited higher or the same positive predictive value (PPV) and negative predictive value (NPV) [21]. Furthermore, a study that aimed at evaluating the effect of bezafibrate compared to UDCA assessed patients after 6 months of drug treatment as well. Therefore, we determined whether a patient was a responder or nonresponder at 6 months after medications were administered [22].

In contrast to our results, one pilot study, where twice the standard dosage was tested, concluded that a double dosage may not benefit the majority of refractory conditions
TABLE 2: Response by definition of Barcelona and Paris-II criteria at 6 months.

\begin{tabular}{lcc}
\hline 6-month & Response rate & $P$ value \\
\hline Paris-II & $13 / 36(36.1 \%)$ & \\
$13-15 \mathrm{mg} / \mathrm{kg} / \mathrm{d}$ & $22 / 37(59.4 \%)$ & 0.046 \\
$\quad 18-22 \mathrm{mg} / \mathrm{kg} / \mathrm{d}$ & $9 / 36(25.0 \%)$ & \\
Barcelona & $12 / 37(32.4 \%)$ & 0.483 \\
$13-15 \mathrm{mg} / \mathrm{kg} / \mathrm{d}$ & \\
$18-22 \mathrm{mg} / \mathrm{kg} / \mathrm{d}$ & & \\
\hline
\end{tabular}

TABLE 3: Response by definition of Barcelona and Paris-II criteria at 12 months.

\begin{tabular}{lcc}
\hline 12-month & Response rate & $P$ value \\
\hline Paris-II & & \\
$13-15 \mathrm{mg} / \mathrm{kg} / \mathrm{d}$ & $17 / 36(47.2 \%)$ & 0.295 \\
$\quad 18-22 \mathrm{mg} / \mathrm{kg} / \mathrm{d}$ & $22 / 37(59.4 \%)$ & \\
Barcelona & & \\
$13-15 \mathrm{mg} / \mathrm{kg} / \mathrm{d}$ & $14 / 36(38.9 \%)$ & 0.401 \\
$18-22 \mathrm{mg} / \mathrm{kg} / \mathrm{d}$ & $18 / 37(48.6 \%)$ & \\
\hline
\end{tabular}

[23]. Nonresponders enrolled in that trial were reported to have been on standard UDCA therapy for 24-141 months [23], which is longer than our patients, and this may be one of the reasons for the difference between our conclusions. However, the administration of high-dose UDCA to earlystage $\mathrm{PBC}$ patients in another trial was found to be positively correlated with the UDCA concentration in bile acids (BAs) and improved liver function [24], which supported our conclusion.

With several clinical trials demonstrating the therapeutic effect of obeticholic acid (OCA) and fibrates, both drugs are now being proposed as second-line treatment options for patients who respond incompletely to or do not tolerate UDCA [25]. Approximately 50\% and 30\% of patients biochemically respond to OCA and fibrates, respectively, with significantly decreased ALP and normalized TB levels [22, 26]. However, side effects occurred more commonly in the treated patients than in the placebo group. Pruritus, the most common complaint of OCA, occurred in more than half of the patients on novel therapy [26]. UDCA, a natural endogenous BA, alleviates cholestasis and reduces damage to cholangiocytes primarily by promoting bile excretion and decreasing BA toxicity [9]. BAs make up an important component of bile, and some are hydrophobic in nature and have toxic effects on the bile duct epithelium. The proportion of UDCA in the bile composition of PBC patients was substantially increased, replacing the toxic $\mathrm{BAs}$ and acting as a cytoprotective agent $[8,27]$. Interestingly, the toxicity of BAs is $\mathrm{pH}$-dependent. UDCA promotes bicarbonate excretion and reduces protonation of BA so that it avoids BA-induced apoptosis [28]. From our result, we can find that adverse reactions were not observed with high-dosage UDCA, which meant the high dosage was well tolerated. Though the gain in terms of biochemical response was not impressive at 12 months, the risk score predicted by the UK-PBC model and the 
TABLE 4: UK-PBC risk score.

\begin{tabular}{lccc}
\hline & $13-15 \mathrm{mg} / \mathrm{kg} / \mathrm{d}(n=36)$ & $18-22 \mathrm{mg} / \mathrm{kg} / \mathrm{d}(n=37)$ & $P$ value \\
\hline UK-PBC-5 year & $0.0257(0.0118,0.0614)$ & $0.0152(0.0100,0.027)$ & 0.006 \\
UK-PBC-10 year & $0.0835(0.0391,0.1912)$ & $0.0533(0.0362,0.0809)$ & 0.024 \\
UK-PBC-15 year & $0.1498(0.0714,0.3263)$ & $0.0943(0.0664,0.1453)$ & 0.025 \\
\hline
\end{tabular}

TABLE 5: Fibrosis risk score testing at the 6th and 12th month.

\begin{tabular}{cccc}
\hline & $13-15 \mathrm{mg} / \mathrm{kg} / \mathrm{d}$ & $18-22 \mathrm{mg} / \mathrm{kg} / \mathrm{d}$ & $P$ value \\
\hline 6 month & & & \\
APRI & $1.016(0.530,2.012)$ & $0.615(0.473,1.135)$ & 0.041 \\
FIB-4 & $2.501(1.550,4.814)$ & $1.729(1.193,2.549)$ & 0.018 \\
12 month & & & $0.731(0.467,1.114)$ \\
APRI & $1.165(0.641,2.038)$ & $2.036(1.343,2.691)$ & 0.008 \\
FIB-4 & $2.874(1.794,4.993)$ & 0.013 \\
\hline
\end{tabular}

Note. APRI, aspartate aminotransferase/platelet ratio index; FIB-4, fibrosis index based on the four factors.

TABle 6: Percentage of biochemical relative changes from baseline at 6 months.

\begin{tabular}{lccc}
\hline \%Change from baseline & $\begin{array}{c}13-15 \mathrm{mg} / \mathrm{kg} / \mathrm{d} \\
(n=36)\end{array}$ & $\begin{array}{c}18-22 \mathrm{mg} / \mathrm{kg} / \mathrm{d} \\
(n=37)\end{array}$ & $P$ value \\
\hline TB, $\mu$ mol/L & $6.79(-12.31,23.09)$ & $9.69(-9.91,26.70)$ & $16.09(4.02,28.12)$ \\
ALP, IU/L & $9.60(-4.03,28.13)$ & $-29.18(19.22,40.03)$ \\
GGT, IU/L & $31.57(7.83,42.76)$ & $15.15(5.00,32.67)$ & 0.544 \\
AST, IU/L & $10.56(-4.80,28.25)$ & $21.28(0.55,39.00)$ & 0.974 \\
ALT, IU/L & $10.71(-4.27,34.31)$ & $1.76(-1.89,6.17)$ & 0.544 \\
ALB, g/L & $-2.21(-8.37,2.97)$ & $2.23(-2.51,9.94)$ & 0.522 \\
GLB, g/L & $2.18(6.19,6.70)$ & $1.72(-12.71,22.83)$ & 0.013 \\
PLT, 10 $/ \mathrm{L}$ & $-9.49(21.55,1.59)$ & $6.67(0.72,15.04)$ & 0.460 \\
IGM, mg/L & $8.36(-5.45,19.09)$ & 0.501 \\
\hline
\end{tabular}

Note. TB, total bilirubin; ALP, alkaline phosphatase; AST, aspartate aminotransferase; ALT, alanine aminotransferase; ALB, albumin; GLB, globulin; GGT, $\gamma$-glutamyltransferase; IGM, immunoglobulin M; PLT, platelet count.

TABle 7: Percentage of biochemical relative changes from baseline at 12 months.

\begin{tabular}{|c|c|c|c|}
\hline$\%$ Change from baseline & $\begin{array}{c}13-15 \mathrm{mg} / \mathrm{kg} / \mathrm{d} \\
(n=36)\end{array}$ & $\begin{array}{c}18-22 \mathrm{mg} / \mathrm{kg} / \mathrm{d} \\
(n=37)\end{array}$ & $P$ value \\
\hline $\mathrm{TB}, \mu \mathrm{mol} / \mathrm{L}$ & $8.30(-18.41,25.40)$ & $2.25(12.84,25.52)$ & 0.860 \\
\hline ALP, IU/L & $19.89(3.68,39.27)$ & $21.16(6.49,34.16)$ & 0.830 \\
\hline GGT, IU/L & $-27.90(-2.25,55.16)$ & $31.86(-18.08,42.67)$ & 0.643 \\
\hline AST, IU/L & $20.42(-12.83,31.50)$ & $23.81(7.31,33.33)$ & 0.277 \\
\hline $\mathrm{ALT}, \mathrm{IU} / \mathrm{L}$ & $20.71(-18.46,39.65)$ & $28.57(9.57,39.29)$ & 0.351 \\
\hline $\mathrm{ALB}, \mathrm{g} / \mathrm{L}$ & $-1.88(-4.96,6.93)$ & $-0.44(-3.52,7.15)$ & 0.354 \\
\hline GLB, g/L & $2.23(-2.37,11.19)$ & $4.05(-1.70,10.62)$ & 0.544 \\
\hline $\mathrm{PLT}, 10^{9} / \mathrm{L}$ & $11.67(-0.49,23.90)$ & $1.72(-18.03,18.86)$ & 0.075 \\
\hline $\mathrm{IGM}, \mathrm{mg} / \mathrm{L}$ & $11.49(-3.17,20.14)$ & $14.05(8.18,30.33)$ & 0.120 \\
\hline
\end{tabular}

Note. TB, total bilirubin; ALP, alkaline phosphatase; AST, aspartate aminotransferase; ALT, alanine aminotransferase; ALB, albumin; GLB, globulin; GGT, $\gamma$-glutamyltransferase; IGM, immunoglobulin M; PLT, platelet count.

noninvasive scores of assessments of fibrosis may also be predictive of a favourable course. Therefore, patients treated with the high UDCA dosage showed some advantages over those who continued the standard dosage in terms of biochemical remission at 6 months and disease progression at 6 and 12 months, indicating that standard therapy with UDCA for 6 months and then another 1 year with high-dosage UDCA for nonresponders may be considered before second-line therapy is recommended.

In this trial, we measured the noninvasive biomarkers APRI and FIB-4 scores to assess the extent of liver fibrosis. The APRI and FIB-4 values have been widely used to 
TABle 8: Adverse reactions through the course of the trial.

\begin{tabular}{lcc}
\hline & $\begin{array}{c}13-15 \mathrm{mg} / \mathrm{kg} / \mathrm{d} \\
(n=36)\end{array}$ & $\begin{array}{c}18-22 \mathrm{mg} / \mathrm{kg} / \mathrm{d} \\
(n=37)\end{array}$ \\
\hline Diarrhea & 0 & 2 \\
Nausea and vomiting & 1 & 1 \\
Rash & 1 & 1 \\
High blood pressure & 1 & 0 \\
\hline
\end{tabular}

predict fibrosis and risk of events $[29,30]$. The UK-PBC scoring system comprehensively integrated relevant prognostic variables and were tested in large, multicenterbased cohorts. Their discriminative ability has been demonstrated to be superior to other existing criteria $[17,31]$. Hence, it is likely that patients in the high-dosage group have less disease progression than those in the standard group.

There are still some limitations of our trial that should be noted. The clinical presentation of patients, such as fatigue and pruritus, was not considered, as fatigue and/or pruritus at onset predict a poorer response to UDCA and prognosis $[32,33]$. Evidence from liver biopsy to provide support from a histological perspective was lacking. Also, there was a potential bias in the evaluation of UK-PBC scores as the presence of cirrhosis seemed to be more frequent in the standard therapy group compared with the high-dose UDCA group, though there was no statistically significant difference.

In conclusion, our study shows that increasing the dosage of UDCA contributes to the therapeutic potency for treatment of $\mathrm{PBC}$ nonresponders, indicating that standard therapy with UDCA for 6 months and then another 1 year with high-dosage UDCA for nonresponders could be a treatment algorithm before second-line therapy is recommended.

\section{Data Availability}

No additional data are available.

\section{Ethical Approval}

The study protocol was in accordance with the Declaration of Helsinki and was approved by the Chinese Ethics Committee (no. ChiECRCT-2017096).

\section{Consent}

All subjects provided written informed consent before enrollment.

\section{Conflicts of Interest}

The authors declare that they have no conflicts of interest.

\section{Authors' Contributions}

Xinyu Xiang and Xiaoli Yang contributed equally to this work.

\section{Acknowledgments}

This work was supported by grants from 1.3.5 project for disciplines of excellence-Clinical Research Incubation Project, West China Hospital, Sichuan University (2019HXFH025).

\section{References}

[1] Z. M. Younossi, D. Bernstein, M. L. Shiffman et al., "Diagnosis and management of primary biliary cholangitis," The American Journal of Gastroenterology, vol. 114, no. 1, pp. 48-63, 2019.

[2] D. Tan and Z. D. Goodman, "Liver biopsy in primary biliary cholangitis," Clinics in Liver Disease, vol. 22, no. 3, pp. 579-588, 2018.

[3] E. Rubin, F. Schaffner, and H. Popper, "Primary biliary cirrhosis. Chronic non-suppurative destructive cholangitis," American Journal of Gastroenterology, vol. 46, pp. 387-407, 1965.

[4] A. J. Montano-Loza and C. Corpechot, "Definition and management of patients with primary biliary cholangitis and an incomplete response to therapy," Clinical Gastroenterology and Hepatology, vol. 20, 2020.

[5] K. Lindor, T. Therneau, R. Jorgensen, M. Malinchoc, and E. Dickson, "Effects of ursodeoxycholic acid on survival in patients with primary biliary cirrhosis," Gastroenterology, vol. 110, no. 5, pp. 1515-1518, 1996.

[6] R. E. Poupon, R. Poupon, and B. Balkau, "Ursodiol for the long-term treatment of primary biliary cirrhosis," New England Journal of Medicine, vol. 330, no. 19, pp. 1342-1347, 1994.

[7] A. Parés, L. Caballería, J. Rodés et al., "Long-term effects of ursodeoxycholic acid in primary biliary cirrhosis: results of a double-blind controlled multicentric trial," Journal of Hepatology, vol. 32, no. 4, pp. 561-566, 2000.

[8] K. Dilger, S. Hohenester, U. Winkler-Budenhofer et al., "Effect of ursodeoxycholic acid on bile acid profiles and intestinal detoxification machinery in primary biliary cirrhosis and health," Journal of Hepatology, vol. 57, pp. 133-140, 2012.

[9] E. J. Carey, A. H. Ali, and K. D. Lindor, "Primary biliary cirrhosis," The Lancet, vol. 386, no. 10003, pp. 1565-1575, 2015.

[10] H. J. F. V. Hoogstraten, M. B. M. D. Smet, W. Renooij et al., "A randomized trial in primary biliary cirrhosis comparing ursodeoxycholic acid in daily doses of either $10 \mathrm{mg} / \mathrm{kg}$ or $20 \mathrm{mg} / \mathrm{kg}$," Alimentary Pharmacology \& Therapeutics, vol. 12, no. 10, pp. 965-971, 1998.

[11] K. D. Lindor, M. E. Gershwin, R. Poupon, M. Kaplan, N. V. Bergasa, and E. J. Heathcote, "Primary biliary cirrhosis," Hepatology, vol. 50, no. 1, pp. 291-308, 2009.

[12] C. Corpechot, O. Chazouillères, and R. Poupon, "Early primary biliary cirrhosis: biochemical response to treatment and prediction of long-term outcome," Journal of Hepatology, vol. 55, no. 6, pp. 1361-1367, 2011.

[13] D. Schuppan and N. H. Afdhal, "Liver cirrhosis," The Lancet, vol. 371, no. 9615, pp. 838-851, 2008.

[14] G. Garcia-Tsao, J. G. Abraldes, A. Berzigotti, and J. Bosch, "Portal hypertensive bleeding in cirrhosis: risk stratification, diagnosis, and management: 2016 practice guidance by the American Association for the study of liver diseases," Hepatology (Baltimore, Md.), vol. 65, pp. 310-335, 2017.

[15] K. D. Lindor, C. L. Bowlus, J. Boyer, C. Levy, and M. Mayo, "Primary biliary cholangitis: 2018 practice guidance from the 
American association for the study of liver diseases," Hepatology (Baltimore, Md.), vol. 69, no. 1, pp. 394-419, 2019.

[16] P. Angulo, E. R. Dickson, T. M. Therneau et al., "Comparison of three doses of ursodeoxycholic acid in the treatment of primary biliary cirrhosis: a randomized trial," Journal of Hepatology, vol. 30, no. 5, pp. 830-835, 1999.

[17] M. Carbone, S. J. Sharp, S. Flack et al., "The UK-PBC risk scores: derivation and validation of a scoring system for longterm prediction of end-stage liver disease in primary biliary cholangitis," Hepatology, vol. 63, no. 3, pp. 930-950, 2016.

[18] A. Vallet-Pichard, V. Mallet, B. Nalpas et al., "FIB-4: an inexpensive and accurate marker of fibrosis in HCV infection. Comparison with liver biopsy and fibrotest," Hepatology, vol. 46, no. 1, pp. 32-36, 2007.

[19] A. Granito, P. Muratori, L. Muratori et al., "Antinuclear antibodies giving the "multiple nuclear dots" or the "rim-like/ membranous" patterns: diagnostic accuracy for primary biliary cirrhosis," Alimentary Pharmacology and Therapeutics, vol. 24, no. 11-12, pp. 1575-1583, 2006.

[20] A. Granito, P. Muratori, C. Quarneti, G. Pappas, R. Cicola, and L. Muratori, "Antinuclear antibodies as ancillary markers in primary biliary cirrhosis," Expert Review of Molecular Diagnostics, vol. 12, no. 1, pp. 65-74, 2012.

[21] L.-N. Zhang, T.-Y. Shi, X.-H. Shi et al., "Early biochemical response to ursodeoxycholic acid and long-term prognosis of primary biliary cirrhosis: results of a 14-year cohort study," Hepatology, vol. 58, no. 1, pp. 264-272, 2013.

[22] C. Corpechot, O. Chazouillères, A. Rousseau et al., "A placebo-controlled trial of bezafibrate in primary biliary cholangitis," New England Journal of Medicine, vol. 378, no. 23, pp. 2171-2181, 2018.

[23] P. Angulo, R. A. Jorgensen, and K. D. Lindor, "Incomplete response to ursodeoxycholic acid in primary biliary cirrhosis: is a double dosage worthwhile?" The American Journal of Gastroenterology, vol. 96, no. 11, pp. 3152-3157, 2001.

[24] E. Roda, F. Azzaroli, G. Nigro et al., "Improved liver tests and greater biliary enrichment with high dose ursodeoxycholic acid in early stage primary biliary cirrhosis," Digestive and Liver Disease, vol. 34, no. 7, pp. 523-527, 2002.

[25] G. M. Hirschfield, U. Beuers, C. Corpechot et al., "EASL Clinical Practice Guidelines: the diagnosis and management of patients with primary biliary cholangitis," Journal of Hepatology, vol. 1, 2017.

[26] F. Nevens, P. Andreone, G. Mazzella et al., "A placebocontrolled trial of obeticholic acid in primary biliary cholangitis," New England Journal of Medicine, vol. 375, no. 7, pp. 631-643, 2016.

[27] B. Terziroli Beretta-Piccoli, G. Mieli-Vergani, D. Vergani et al., "The challenges of primary biliary cholangitis: what is new and what needs to be done," Journal of Autoimmunity, vol. 105, Article ID 102328, 2019.

[28] U. Beuers, S. Hohenester, L. J. M. de Buy Wenniger, A. E. Kremer, P. L. M. Jansen, and R. P. J. O. Elferink, "The biliary $\mathrm{HCO}_{3}$ - umbrella: a unifying hypothesis on pathogenetic and therapeutic aspects of fibrosing cholangiopathies," Hepatology, vol. 52, no. 4, pp. 1489-1496, 2010.

[29] P. J. Trivedi, T. Bruns, A. Cheung et al., "Optimising risk stratification in primary biliary cirrhosis: AST/platelet ratio index predicts outcome independent of ursodeoxycholic acid response," Journal of Hepatology, vol. 60, no. 6, pp. 1249-1258, 2014.

[30] A. Unalp-Arida and C. E. Ruhl, "Liver fibrosis scores predict liver disease mortality in the United States population," Hepatology, vol. 66, no. 1, pp. 84-95, 2017.
[31] W. J. Lammers, G. M. Hirschfield, C. Corpechot et al., "Development and validation of a scoring system to predict outcomes of patients with primary biliary cirrhosis receiving ursodeoxycholic acid therapy," Gastroenterology, vol. 149, no. 7, pp. 1804-1812, Article ID e1804, 2015.

[32] C. Quarneti, P. Muratori, C. Lalanne et al., "Fatigue and pruritus at onset identify a more aggressive subset of primary biliary cirrhosis," Liver International, vol. 35, no. 2, pp. 636-641, 2015.

[33] D. E. Jones, A. Al-Rifai, J. Frith, I. Patanwala, and J. L. Newton, "The independent effects of fatigue and UDCA therapy on mortality in primary biliary cirrhosis: results of a 9 year follow-up," Journal of Hepatology, vol. 53, no. 5, pp. 911-917, 2010. 